\title{
Comparing Cerebral Palsy Surveillance Definition to ICD Codes and Written Diagnoses
}

\section{Sarabeth Mathis, Matthew Maenner, Lucinda England, Fatuma Abdirizak, Kim Van Naarden Braun, Deborah Christensen, Nicole Dowling, Maureen Durkin ${ }^{4}$, Robert Fitzgerald 5 , Russell Kirby $^{6}$, Laura Schieve ${ }^{1}$, Marshalyn Yeargin-Allsopp ${ }^{1}$, Patricia Dietz ${ }^{1}$}

\footnotetext{
${ }^{1}$ National Center on Birth Defects \& Developmental Disabilities, Centers for Disease Control and Prevention, Atlanta, Georgia, United States, ${ }^{2}$ Oak Ridge Institute for Science and Education, Oak Ridge, Tennessee, United States, ${ }^{3}$ Hackensack Meridian Health, Edison, Georgia, United States, ${ }^{4}$ University of Wisconsin- Madison, Madison, Wisconsin, United States, ${ }^{5}$ Washington University in St Louis, St Louis, Missouri, United States,

${ }^{6}$ University of South Florida, Tampa, Florida, United States
}

\section{Objective}

To compare prevalence estimates obtained by the ADDM cerebral palsy surveillance method to other administrative or diagnostic indications of cerebral palsy.

\section{Introduction}

Cerebral Palsy (CP) is the most common cause of motor disability in children. CP registries often rely on administrative data such as CP diagnoses or International Classification of Diseases (ICD) codes indicative of CP. However, little is known about the validity of these indicators. We calculated sensitivity, specificity, positive and negative predictive values of CP ICD-9 codes and CP diagnoses compared to a "gold standard" CP classification based on detailed medical and education record review.

\section{Methods}

This sample includes 50,332 8-year-olds living in four US sites (32 counties in Alabama, 5 counties in Georgia, 10 counties in Wisconsin, and 5 counties in Missouri) in 2006, 2008, and 2010. The Autism and Developmental Disabilities Monitoring (ADDM) Network reviewed medical and education records for these children as part of the US Centers for Disease Control and Prevention population-based surveillance of developmental disabilities. All of these children received special education services or were assigned one or more ICD-9 codes associated with a variety of developmental disabilities by community medical providers.

Medical and education records were reviewed by trained staff; if the records contained CP diagnoses or motor findings indicative of $\mathrm{CP}$, detailed clinical information was abstracted for additional review by trained clinicians who determined whether the child met the $\mathrm{CP}$ case definition based on all information available. Abstracted records were also reviewed for evidence of known motor disorders or genetic conditions that disqualified a child from being a CP case, such as inborn error of metabolism or muscular dystrophy. Trained clinicians reviewed and excluded children with confirmed disqualifying conditions.

We calculated CP prevalence, sensitivity, specificity, and positive and negative predictive values for three different methods used to identify cases, using the ADDM surveillance case identification as the gold standard. These methods include: 1) ICD-9 codes for $\mathrm{CP}(342-344) ; 2)$ a CP diagnosis written in the medical or education records, excluding children with disqualifying conditions, and 3) both ICD-9 codes (342-344) and a CP diagnosis written in the medical or education records, excluding children with disqualifying conditions. In an attempt to avoid requiring record review for method 1, we considered using ICD-9 codes for disqualifying conditions. However, we found that ICD codes for these conditions did not correlate well with disqualifying conditions identified in medical record reviews; therefore disqualifying conditions were not considered for method 1 . Methods 2 and 3 did require review of medical records for disqualifying conditions and for a written CP diagnosis, but overall were less extensive than traditional ADDM surveillance methods.

In order to determine the impact of different classification criteria on how and which children are captured by surveillance methods, we compared demographic and other characteristics of all children who met the ADDM surveillance case definition. We compared children who would and would not be classified as CP cases using method 3.

SDS Annual Conference Proceedings 2019. This is an Open Access article distributed under the terms of the Creative Commons AttributionNoncommercial 4.0 Unported License (http://creativecommons.org/licenses/by-nc/3.0/), permitting all non-commercial use, distribution, and reproduction in any medium, provided the original work is properly cited. 


\section{Results}

Out of the total 50,332 children, 1294 met the ADDM surveillance case definition, 2201 had CP ICD codes (method 1), 1502 had a written CP diagnosis and no disqualifying conditions (method 2), and 1345 had both CP ICD codes and a written diagnosis and no disqualifying conditions (method 3). Each study year, between $32-48 \%$ of abstracted children were excluded due to disqualifying conditions found in medical records. The ADDM network gold standard CP prevalence was 3.3 per 1000 in 2006 , 3.1 per 1000 in 2008 , and 2.9 per 1000 in 2010 .

For method 1, sensitivity was $90.0 \%$, specificity was $97.4 \%$, positive predictive value was $51.6 \%$ and negative predictive value was $99.7 \%$. Method 1 prevalence estimates were 5.3 per 1000 in 2006, 4.6 per 1000 in 2008, and 4.6 per 1000 in 2010 . For method 2, sensitivity was $98.1 \%$, specificity was $88.4 \%$, PPV was $84.5 \%$ and NPV was $98.4 \%$ compared to the ADDM Network definition. Method 2 estimated prevalence was 3.9 per 1000 for 2006, 3.6 per 1000 for 2008, and 3.2 per 1000 for 2010. For method 3, sensitivity was $89.6 \%$, specificity was $99.5 \%$, PPV was $84.3 \%$ and NPV was $99.7 \%$. Method 3 estimated prevalence was 3.5 per 1000 for 2006, 3.2 per 1000 for 2008, and 2.8 per 1000 for 2010.

Using Pearson's Chi-Square tests, we compared demographic and other characteristics of ADDM Network CP case children who also met method 3 case definition $(n=1134)$ and children who met the ADDM Network CP definition but not method 3 case definition $(\mathrm{n}=160)$. Demographic information was not different between these children. ADDM Network CP case children who did not meet method 3 criteria were significantly less likely to require a wheelchair for mobility than children who met method 3 criteria $(4.4 \%$ versus $27.4 \%, \mathrm{p}<.05)$.

\section{Conclusions}

Relying on ICD-9 codes without excluding disqualifying conditions to identify CP cases (method 1) resulted in high sensitivity (90\%), but low positive predictive value as well as an overestimated CP prevalence when compared with the ADDM Network method. Use of a written diagnosis and excluding disqualifying conditions (method 2) resulted in very high sensitivity (98\%), with fewer false positives but overestimated CP prevalence compared to the ADDM estimate. In contrast, using both CP ICD codes and a written CP diagnosis and excluding disqualifying conditions (method 3) yielded prevalence estimates similar to ADDM Network $\mathrm{CP}$ estimates; this approach also had high sensitivity, specificity, and PPV. Methods 2 and 3 still require manual record review, unlike method 1. For method 2, reviewers would need to review all records for $\mathrm{CP}$ and disqualifying conditions. Method 3 only requires review of records with CP ICD codes, comprising 4\% of all records currently reviewed. Method 3 would fail to capture children without both a written diagnosis and ICD codes; and this approach may be less sensitive for detecting CP among children with less severe motor impairment than using the gold standard.

Using ICD codes and written CP diagnoses contained in medical and education records combined with a limited medical record review to identify disqualifying conditions could lower operational costs of CP surveillance while preserving accurate prevalence estimates compared with the more labor-intensive processes currently used. Further evaluation is needed to determine if improvements in efficiency are worth potential trade-offs in the data collected by the system. Of particular importance is whether the approach could capture all the necessary indicators that are important to stakeholders. Additional analyses would also need to evaluate whether the surveillance methods affect other findings, such as previously observed disparities, co-occurring conditions, or CP severity.

\section{Acknowledgement}

This project was supported in part by an appointment to the Research Participation Program at the Centers for Disease Control and Prevention administered by the Oak Ridge Institute for Science and Education through an interagency agreement between the U.S. Department of Energy and the Centers for Disease Control and Prevention 